\title{
SHADOW LIMIT FOR PARABOLIC-ODE SYSTEMS THROUGH A CUT-OFF ARGUMENT
}

\author{
Anna Marciniak-Czochra and Andro Mikelić
}

This contribution is dedicated to the memory of Professor Sibe Mardešić and his professional life dedicated to the development of the mathematical sciences in Croatia.

AbSTRACT. We study a shadow limit (the infinite diffusion coefficientlimit) of a system of ODEs coupled with a diagonal system of semilinear heat equations in a bounded domain with homogeneous Neumann boundary conditions. The recent convergence proof by the energy approach from [19], developed for the case of a single PDE, is revisited and generalized to the case of the coupled system. Furthermore, we give a new convergence proof relying on the introduction of a well-prepared related cut-off system and on a construction of the barrier functions and comparison test functions, new in the literature. It leads to the $L^{\infty}$-estimates proportional to the inverse of the diffusion coefficient.

\section{INTRODUCTION}

In the study of coupled systems of evolution differential equations describing Turing-type pattern formation, the diffusion coefficients are typically of very different orders of magnitude (see [7], [5], [14], [20] and references therein).

The spatio-temporal evolution of solutions of such models is considered in numerous applied articles and if one could reduce the system by taking an infinite diffusion limit, the task would get much simpler. The reduction through the infinite diffusion limit has been employed in various biological contexts, see e. g. [10,12,17,18].

A pioneering rigorous study of such limit is in reference [15] by MarciniakCzochra and collaborators. An approach using semigroup convergence has been recently undertaken by Bobrowski in ref. [1]. They established the convergence for finite times but did not study error estimates.

A detailed formal derivation of the reduced systems in such limit, using the renormalization group technique was undertaken in [19]. In addition

2010 Mathematics Subject Classification. 35B20, 34E13, 35B25, 35B41, 35K57.

Key words and phrases. Shadow limit, reaction-diffusion equations, model reduction. 
rigorous proofs of a large diffusion limit for such models, through an energy estimate and through the center manifold approach were given. In this paper we focus on the energy estimate and obtain the optimal bounds through a study of an associated auxiliary system, where the nonlinearities are cut-off. Also we generalize the study of a single semilinear heat equations, coupled with a system of ODEs, from [19], to the case of a system of semilinear parabolic equations coupled with a system of ODEs. We point out that in [19] the standard comparison functions from the $L^{\infty}$-estimates for the scalar parabolic equations were sufficient to get the optimal $L^{\infty}$-bounds. In the case of semilinear parabolic systems, it is not the case any more. If the diffusion coefficient is $1 / \varepsilon$, using the classical comparison functions as in [13], chapter 6 , leads to $L^{\infty}$-error estimates of order $O(\sqrt{\varepsilon})$, which is not optimal. Hence, we present a new construction of the barrier functions and of the comparison test functions, different from [13], chapter 6 , and leading to the optimal $L^{\infty}$-error estimates of order $O(\varepsilon)$.

To be more precise, we consider an ODE system, coupled with a diagonal system of semilinear parabolic equations with a large diffusion coefficient. Its ratio to the other coefficients is equal to the inverse of a small parameter $\varepsilon>0$. We assume that $\Omega$ is a given open bounded set in $\mathbb{R}^{n}, 1 \leq n \leq 3$, with a smooth boundary and focus on the Cauchy problem

$$
\begin{aligned}
\frac{\partial \mathbf{u}_{\varepsilon}}{\partial t} & =\mathbf{f}\left(\mathbf{u}_{\varepsilon}, \mathbf{v}_{\varepsilon}\right), \quad \text { in } \quad(0, T) \times \Omega, \\
\frac{\partial \mathbf{v}_{\varepsilon}}{\partial t} & =\frac{1}{\varepsilon} \Delta \mathbf{v}_{\varepsilon}+\Phi\left(\mathbf{u}_{\varepsilon}, \mathbf{v}_{\varepsilon}\right), \text { in }(0, T) \times \Omega, \\
\frac{\partial \mathbf{v}_{\varepsilon}}{\partial \nu} & =0 \text { in }(0, T) \times \partial \Omega, \\
\mathbf{u}_{\varepsilon}(0) & =\mathbf{u}^{0}(x) \text { and } \mathbf{v}_{\varepsilon}(0)=\mathbf{v}^{0}(x), x \in \Omega .
\end{aligned}
$$

We set $Q_{T}=\Omega \times(0, T)$.

Asymptotic analysis of problem (1.1)-(1.4) with $\varepsilon \rightarrow 0$ has attracted a considerable interest in the literature in the case where the first equation is a quasilinear parabolic equation, starting from the papers of Keener [11] and Hale [8]. Our setting is different.

The calculations from [19] using the renormalization group analysis (see [3], [4] or [6]), permit the shadow limit $\varepsilon \rightarrow+0$ reduction of equations (1.1)(1.4). They yield the following system of integro-differential equations

$$
\begin{aligned}
& \frac{\partial \mathbf{u}}{\partial t}=\mathbf{f}(\mathbf{u}, \mathbf{v}), \quad \text { in } \quad(0, T) \times \Omega \\
& \frac{d \mathbf{v}}{d t}=\frac{1}{|\Omega|} \int_{\Omega} \Phi(\mathbf{u}(x, t), \mathbf{v}(t)) d x, \quad \text { in } \quad(0, T)
\end{aligned}
$$


In this article we present a detailed study of the limit process by comparing solutions of the two systems (1.1)-(1.4) and (1.5)-(1.6) and proving an error estimate in terms of $\varepsilon$. The employed methods are (i) the introduction of a cut-off problem, (ii) the maximum principle calculations for the parabolic systems coupled with ODEs and (iii) the energy estimates. The novelty in comparison with [19] is in (i) considering a system of semilinear parabolic PDEs, (ii) in studying the asymptotic behavior as $\varepsilon \rightarrow 0$ of the cutoff problem instead of problem (1.1)-(1.4) and (iii) in providing self-contained well-posedness proofs for the shadow system (1.5)-(1.6) and for the cut-off problem (2.36)-(2.39). As explained above, passing from the scalar semilinear parabolic equation to a semilinear parabolic system required a new comparison argument.

The results are in section 2. Subsection 2.1 is dedicated to the wellposedness of the shadow limit problem (1.5)-(1.6) in its nonhomogeneous version. Subsection 2.2 contains a short presentation of two auxiliary problems, correcting the reduction of the initial condition to its arithmetic mean and the parabolic nonlinearities to their arithmetic means. The study of the $\varepsilon$-problem (1.1)-(1.4) is replaced in subsection 2.3 by the study of a well-prepared cutoff problem. Its well-posedness is studied in details. Subsection 2.4 contains crucial $L^{\infty}$-estimates in proposition 2.6. They allow concluding that the solution to the cut-off problem leads to the solution of the $\varepsilon$-problem (1.1)-(1.4). In subsection 2.5 the $L^{\infty}$ error estimate of order $\varepsilon$, for the difference of the solution between the $\varepsilon$-problem and the shadow limit problem is given. In Theorem 2.10 it is completed with an $O\left(\varepsilon^{3 / 2}\right)$ energy estimate in $H^{1}$, for the fluctuation around the mean of the solutions to the parabolic part of the $\varepsilon$-problem.

\section{The Shadow Limit Through A CUT-OFF PRoblem AND THE ENERGy AND $L^{\infty}$-ESTIMATES}

We consider the asymptotic behavior as $\varepsilon \rightarrow 0$ of the Cauchy' problem (1.1)-(1.4). The nonlinearities $\mathbf{f}$ and $\Phi$ are defined on $\mathbb{R}^{m+k}, m, k \geq 1$, and take values in $\mathbb{R}^{m}$ and $\mathbb{R}^{k}$, respectively. For simplicity, we will denote the arithmetic mean by

$$
\langle z\rangle_{\Omega}=\frac{1}{|\Omega|} \int_{\Omega} z(x) d x, \quad z \in L^{1}(\Omega) .
$$

We proceed in several steps, achieved in separate subsections. 
2.1. Existence results for the shadow problem. We start by establishing properties of the slightly more general shadow system (1.5)-(1.6):

$$
\begin{aligned}
\frac{\partial \mathbf{A}}{\partial t}=\mathbf{f}(\mathbf{A}, \mathbf{B}, x, t) \text { on } \Omega \times(0, T) ; & \mathbf{A}(x, 0)=\mathbf{u}^{0}(x) \text { in } \Omega ; \\
\frac{d \mathbf{B}}{d t}=\langle\Phi(\mathbf{A}, \mathbf{B}, x, t)\rangle_{\Omega} \text { on }(0, T) ; & \mathbf{B}(0)=\left\langle\mathbf{v}^{0}\right\rangle_{\Omega},
\end{aligned}
$$

where $\Omega$ is a bounded open set in $\mathbb{R}^{n}$, with a $C^{2}$ boundary.

Let $\mathcal{B}(\Omega)$ be a vector space of all functions defined everywhere on $\Omega$ that are bounded and measurable over $\Omega$. $\mathcal{B}(\Omega)$ becomes a Banach space when equipped with the norm $\|g\|_{\mathcal{B}(\Omega)}=\sup _{x \in \Omega}|g(x)|$. We make the following

\section{Assumptions:}

A1. $\mathbf{f}$ is a $C^{1}$ function of $x \in \bar{\Omega}$ and a continuous function of $t \in[0, T]$. $\Phi$ is a continuous function in $(x, t) \in \bar{\Omega} \times[0, T]$.

A2. $\mathbf{f}$ and $\Phi$ are $C^{\infty}$ functions in $(\mathbf{A}, \mathbf{B}) \in \mathbb{R}^{m+k}$, where $m$ and $k$ are integers bigger or equal than 1 .

A3. $\mathbf{u}^{0} \in \mathcal{B}(\Omega)^{m}, \mathbf{v}^{0} \in L^{1}(\Omega)^{k}$.

The well-posedness of the Cauchy problem (2.1)-(2.2) follows from the general theory contained in the textbook of Henry [9] or from the results from ref. [16]. For the comfort of the reader we give an elementary direct proof by applying Picard iteration to our infinite dimensional setting. It yields

TheOREM 2.1. Under Assumptions A1-A3, there is a constant $T_{0}>0$ such that problem (2.1)-(2.2) has a unique solution $\{\mathbf{A}, \mathbf{B}\} \in C^{1}\left(\left[-T_{0}, T_{0}\right]\right.$, $\left.\mathcal{B}(\Omega)^{m} \times \mathbb{R}^{k}\right)$.

Proof. Let $r_{0}>0$ be given. Let $\mathbf{y}_{0}=\left\{\mathbf{u}^{0},\left\langle\mathbf{v}^{0}\right\rangle_{\Omega}\right\}$ and $T$ a positive constant. We set $\bar{B}\left(\mathbf{y}_{0}, r_{0}\right)=\left\{\mathbf{y} \in \mathcal{B}(\Omega)^{m} \times \mathbb{R}^{k} \mid\left\|\mathbf{y}-\mathbf{y}_{0}\right\|_{\mathcal{B}(\Omega)^{m} \times \mathbb{R}^{k}} \leq r_{0}\right\}$ and introduce a cylinder $\mathcal{C}=[-T, T] \times \bar{B}\left(\mathbf{y}_{0}, r_{0}\right)=\{-T \leq t \leq T$ and $\left.\mathbf{y}(t) \in \mathcal{B}(\Omega)^{m} \times \mathbb{R}^{k} \mid\left\|\mathbf{y}(t)-\mathbf{y}_{0}\right\|_{\mathcal{B}(\Omega)^{m} \times \mathbb{R}^{k}} \leq r_{0}\right\}$. We denote the Lipschitz constants, with respect to $\{\mathbf{A}, \mathbf{B}\}$, for $\mathbf{f}$ and $\Phi$ on the cylinder $\mathcal{C}$ by $M_{f}$ and $M_{\phi}$, respectively. The cylinder $\mathcal{C}$, equipped with the topology of the space $C\left([-T, T] ; \mathcal{B}(\Omega)^{m} \times \mathbb{R}^{k}\right)$ is a complete metric space.

We study existence of fixed points for the nonlinear mapping $G=$ $\left\{G_{1}, G_{2}\right\}$, defined on the cylinder $\mathcal{C}$ by

$$
\begin{gathered}
G_{1}(X, Y)=\mathbf{u}^{0}+\int_{0}^{t} \mathbf{f}(X, Y, x, \tau) d \tau, \quad t \in[0, T] \text { and } x \in \Omega, \\
G_{2}(X, Y)=\left\langle\mathbf{v}^{0}\right\rangle_{\Omega}+\int_{0}^{t}\langle\Phi(X, Y, \cdot, \tau)\rangle_{\Omega} d \tau, \quad t \in[0, T]
\end{gathered}
$$

First, let us prove that $G$ maps the cylinder $\mathcal{C}$ into itself, for $T \leq T_{0}$. 
Let

$$
\begin{aligned}
B_{0} & =\sup _{(x, \tau) \in \Omega \times(-T, T)}\left|\mathbf{f}\left(\mathbf{u}^{0}(x),\left\langle\mathbf{v}^{0}\right\rangle_{\Omega}, x, \tau\right)\right| \text { and } \\
F_{0} & =\sup _{(x, \tau) \in \Omega \times(-T, T)}\left|\left\langle\Phi\left(\mathbf{u}^{0}(x),\left\langle\mathbf{v}^{0}\right\rangle_{\Omega}, x, \tau\right)\right\rangle_{\Omega}\right| .
\end{aligned}
$$

Then we have

$$
\begin{gathered}
\left|G_{1}(X, Y)-\mathbf{u}^{0}\right|(x, t)=\left|\int_{0}^{t} \mathbf{f}(X, Y, x, \tau) d \tau\right| \\
\leq t B_{0}+M_{f} \int_{0}^{t}\left(\left|X(x, \tau)-\mathbf{u}^{0}(x)\right|+\left|Y(\tau)-\left\langle\mathbf{v}^{0}\right\rangle_{\Omega}\right|\right) d \tau \leq t\left(B_{0}+M_{f} r_{0}\right), \\
\left|G_{2}(X, Y)-\left\langle\mathbf{v}^{0}\right\rangle_{\Omega}\right|(t)=\left|\int_{0}^{t}\langle\Phi(X, Y, \cdot, \tau)\rangle_{\Omega} d \tau\right| \\
\leq t F_{0}+M_{\Phi} \int_{0}^{t}\left(\left|X(x, \tau)-\mathbf{u}^{0}(x)\right|+\left|Y(\tau)-\left\langle\mathbf{v}^{0}\right\rangle_{\Omega}\right|\right) d \tau \leq t\left(F_{0}+M_{\Phi} r_{0}\right) .
\end{gathered}
$$

The above calculations yield

$$
\left\|G(X, Y)-\left\{\mathbf{u}^{0},\left\langle\mathbf{v}^{0}\right\rangle_{\Omega}\right\}\right\|_{\mathcal{C}} \leq T\left(F_{0}+B_{0}+\left(M_{\Phi}+M_{f}\right) r_{0}\right)
$$

and $G$ maps $\mathcal{C}$ into itself for $T \leq r_{0} /\left(F_{0}+B_{0}+\left(M_{\Phi}+M_{f}\right) r_{0}\right)$.

Next we prove that $G$ is a contraction map for sufficiently small $T$.

$$
\begin{gathered}
\left|G_{1}\left(X_{1}, Y_{1}\right)-G_{1}\left(X_{2}, Y_{2}\right)\right|(x, t)=\left|\int_{0}^{t}\left(\mathbf{f}\left(X_{1}, Y_{1}, x, \tau\right)-\mathbf{f}\left(X_{2}, Y_{2}, x, \tau\right)\right) d \tau\right| \\
\leq M_{f} \int_{0}^{t}\left(\left|X_{1}(x, \tau)-X_{2}(x, \tau)\right|+\left|Y_{1}(\tau)-Y_{2}(\tau)\right|\right) d \tau \\
\leq t M_{f}||\left\{X_{1}, Y_{1}\right\}-\left\{X_{2}, Y_{2}\right\}||_{\mathcal{C}} \\
\left|G_{2}\left(X_{1}, Y_{1}\right)-G_{2}\left(X_{2}, Y_{2}\right)\right|(t)=\left|\int_{0}^{t}\left(\left\langle\Phi\left(X_{1}, Y_{1}, \cdot, \tau\right)\right\rangle_{\Omega}-\left\langle\Phi\left(X_{2}, Y_{2}, \cdot, \tau\right)\right\rangle_{\Omega}\right) d \tau\right| \\
\leq t M_{\Phi}||\left\{X_{1}, Y_{1}\right\}-\left\{X_{2}, Y_{2}\right\} \|_{\mathcal{C}} .
\end{gathered}
$$

Consequently we have

$$
\left.\| G\left(X_{1}, Y_{1}\right)-G\left(X_{2}, Y_{2}\right)\right\}\left\|_{\mathcal{C}} \leq T\left(M_{f}+M_{\Phi}\right)\right\|\left\{X_{1}, Y_{1}\right\}-\left(X_{2}, Y_{2}\right\} \|_{\mathcal{C}}
$$

and for $T<1 /\left(M_{f}+M_{\Phi}\right)$ the map $G$ is a contraction. Note that diminishing the time interval can only make smaller the Lipschitz constants $M_{f}$ and $M_{\Phi}$. The existence and uniqueness of a solution to system (2.1)-(2.2) is now a consequence of the Banach fixed point theorem.

Regularity with respect to the space is not restricted to $\mathcal{B}(\Omega)$. The analogous result with $\mathcal{B}(\Omega)$ replaced by $C(\bar{\Omega})$, holds if we assume $\mathbf{u}^{0} \in C(\bar{\Omega})^{m}$.

Differentiability properties can be shown along the same lines. 
Proposition 2.2. Suppose Assumptions A1.-A3. Then if in addition $\mathbf{u}^{0} \in W^{1, r}(\Omega)^{m}, 1 \leq r<+\infty$, there is a constant $T_{0}>0$ such that problem (2.1)-(2.2) has a unique solution $\{\mathbf{A}, B\} \in C^{1}\left(\left[-T_{0}, T_{0}\right],\left(W^{1, r}(\Omega)^{m} \cap\right.\right.$ $\left.\left.\mathcal{B}(\Omega)^{m}\right) \times \mathbb{R}^{k}\right)$.

Proof. For $j=1, \ldots, n$, the partial derivative $\frac{\partial \mathbf{A}}{\partial x_{j}}$ satisfies the linear system of ordinary differential equations

$$
\begin{gathered}
\partial_{t} \frac{\partial \mathbf{A}}{\partial x_{j}}=\nabla_{A} \mathbf{f} \frac{\partial \mathbf{A}}{\partial x_{j}}+\frac{\partial \mathbf{f}}{\partial x_{j}} \quad \text { on } \quad\left(0, T_{0}\right) \times \Omega, \\
\left.\frac{\partial \mathbf{A}}{\partial x_{j}}\right|_{t=0}=\frac{\partial \mathbf{u}^{0}}{\partial x_{j}} \in L^{r}(\Omega) .
\end{gathered}
$$

Since $\nabla_{A} \mathbf{f} \in L^{\infty}\left(\left(-T_{0}, T_{0}\right) \times \Omega\right)$ and $\frac{\partial \mathbf{f}}{\partial x_{j}} \in L^{\infty}\left(\left(-T_{0}, T_{0}\right) \times \Omega\right)$, the theory of linear ordinary differential equations with bounded coefficients yields $\frac{\partial \mathbf{A}}{\partial x_{j}} \in$ $C^{1}\left(\left[-T_{0}, T_{0}\right], L^{r}(\Omega)^{m} \times \mathbb{R}^{k}\right)$.

If, in addition to Assumptions A1.-A3., we suppose

A4. There exist continuous functions $c, k$, defined on $\mathbb{R}$ with values in $\mathbb{R}_{+}$, such that

$$
\|\left.\mathbf{f}(\mathbf{y}, \cdot, t)\right|_{H^{1}(\Omega)^{m}}+\left|\langle\Phi(\mathbf{y}, \cdot, t)\rangle_{\Omega}\right| \leq c(t)+k(t)|\mathbf{y}|_{\mathbb{R}^{m+k}}, \quad \forall \mathbf{y} \in \mathbb{R}^{m+k},
$$

then every maximal solution to problem (2.1)-(2.2) is global.

2.2. Auxiliary problems corresponding to the initial layer and the decaying oscillations around the mean.

In the following we introduce two auxiliary problems.

The first problem is linked to the fact that in the shadow limit equation, only the mean of $\Phi$ appears. We have to correct the replacement of $\Phi$ by its spatial mean, which is a bad approximation and only the fast decay in time allows handling it. The correction $\mathbf{w}^{\Phi}$ is introduced by

$$
\begin{gathered}
-\Delta_{x} \mathbf{w}^{\Phi}=\Phi(\mathbf{A}, \mathbf{B}, x, t)-\langle\Phi(\mathbf{A}, \mathbf{B}, x, t)\rangle_{\Omega} \text { in } \Omega, \\
\frac{\partial \mathbf{w}^{\Phi}}{\partial \nu}=0 \quad \text { on } \partial \Omega, \quad\left\langle\mathbf{w}^{\Phi}\right\rangle_{\Omega}=0 .
\end{gathered}
$$

We suppose Assumptions A1.-A3. and $\mathbf{u}^{0} \in W^{1, r}(\Omega)^{k}, 1<r<+\infty$. Then problem (2.10)-(2.11) admits a unique variational solution $\mathbf{w}^{\Phi} \in$ $C\left([0, T] ; H^{1}(\Omega)^{k}\right)$. Furthermore, $\mathbf{w}^{\Phi} \in C\left([0, T] ; W^{2, r}(\Omega)^{k}\right)$, for all $r \in$ $[1,+\infty)$. 
Next, using the regularity of $(\mathbf{A}, \mathbf{B})$ established in Theorem 2.1 and Proposition 2.2, we find out that $\partial_{t} \mathbf{w}^{\Phi}$ satisfies

$$
\begin{array}{r}
-\Delta_{x} \partial_{t} \mathbf{w}^{\Phi}=\nabla_{A} \Phi(\mathbf{A}, \mathbf{B}, x, t) \partial_{t} \mathbf{A}+\nabla_{B} \Phi(\mathbf{A}, \mathbf{B}, x, t) \partial_{t} \mathbf{B}+ \\
\partial_{t} \Phi(\mathbf{A}, \mathbf{B}, x, t)-\left\langle\nabla_{A} \Phi(\mathbf{A}, \mathbf{B}, x, t) \partial_{t} \mathbf{A}\right\rangle_{\Omega}- \\
\left\langle\nabla_{B} \Phi(\mathbf{A}, \mathbf{B}, x, t) \partial_{t} \mathbf{B}\right\rangle_{\Omega}-\left\langle\partial_{t} \Phi(\mathbf{A}, \mathbf{B}, x, t)\right\rangle_{\Omega} \in C\left([0, T] ; L^{\infty}(\Omega)\right) ; \\
\frac{\partial}{\partial \nu} \partial_{t} \mathbf{w}^{\Phi}=0 \quad \text { on } \quad \partial \Omega, \quad\left\langle\partial_{t} \mathbf{w}^{\Phi}\right\rangle_{\Omega}=0 .
\end{array}
$$

Therefore

$$
\partial_{t} \mathbf{w}^{\Phi} \in C\left([0, T] ; W^{2, r}(\Omega)^{k}\right), \quad \forall r \in[1,+\infty) .
$$

The second problem is linked to the fact that the shadow approximation uses only the space average of the initial value $\mathbf{v}^{0}$ of $\mathbf{v}$. It creates an initial time layer given by

$$
\left(\frac{\partial}{\partial \tau}-\Delta_{x}\right) \xi^{i}(x, \tau)=0 \quad \text { in } \quad \Omega \times(0,+\infty),
$$

$$
\frac{\partial \xi^{i}}{\partial \nu}=0 \text { on } \partial \Omega \times(0,+\infty), \xi^{i}(x, 0)=\mathbf{v}^{0}(x)-\varepsilon \mathbf{w}^{\Phi}(x, 0)-\left\langle\mathbf{v}^{0}\right\rangle_{\Omega} \text { in } \Omega .
$$

In addition to Assumptions A1.- A2. we suppose

A5. $\mathbf{v}^{0} \in W^{2-2 / r, r}(\Omega)^{m}$ and $\mathbf{u}^{0} \in W^{1, r}(\Omega)^{k}, r>\max \{2,(n+2) / 2\}$.

Then the separation of variables formula for the heat equation yields

$$
\xi^{i}(x, \tau)=\sum_{j=1}^{\infty} e^{-\lambda_{j} \tau}\left(\tilde{\mathbf{v}}^{0}, w_{j}\right)_{L^{2}(\Omega)} w_{j}(x) \in L^{2}\left(0, T ; H^{1}(\Omega)\right) \cap C\left([0, T] ; L^{2}(\Omega)\right),
$$

for all finite positive $T$, with $\tilde{\mathbf{v}}^{0}=\mathbf{v}^{0}-\varepsilon \mathbf{w}^{\Phi}(x, 0)-\left\langle\mathbf{v}^{0}\right\rangle_{\Omega} . \quad\left\{w_{j}\right\}_{j \in \mathbb{N}}$ is the spectral orthonormal basis for $L^{2}(\Omega)$ and an orthogonal basis for $H^{1}(\Omega)$ given by

$$
-\Delta w=\lambda w \quad \text { in } \quad \Omega ; \quad \frac{\partial w}{\partial \nu}=0 \quad \text { on } \quad \partial \Omega .
$$

Furthermore, by the maximum principle for the heat equation $\tilde{\mathbf{v}}^{0} \in L^{\infty}(\Omega)^{k}$ implies $\xi^{i} \in L^{\infty}(\Omega \times(0, T))^{k}$. Note that $\tilde{\mathbf{v}}^{0} \in H^{1}(\Omega)^{k}$ implies $\partial_{t} \xi^{i} \in L^{2}(\Omega \times$ $(0, T))^{k}$ and $\xi^{i} \in L^{\infty}\left(0, T ; H^{1}(\Omega)\right)^{k}$. Finally, we recall that after [13], chapter 4, section $9, \partial \Omega \in C^{2}$ and $\tilde{\mathbf{v}}^{0} \in W^{2, r}(\Omega)^{k},(n+2) / 2<r<+\infty$, imply $\xi^{i} \in W_{r}^{2,1}\left(Q_{T}\right)^{k}$ and it is a Hölder continuous function in $x$ and $t$.

In the remainder of this section, we use the initial layer function $\xi^{i, \varepsilon}(x, t)=\xi^{i}\left(x, \frac{t}{\varepsilon}\right)$. Note that for $\tilde{\mathbf{v}}^{0} \in W^{2, r}(\Omega)^{k}, r>(n+2) / 2$

$$
\left|\xi^{i, \varepsilon}(x, t)\right| \leq C e^{-\lambda_{1} t / \varepsilon} \quad \text { on } Q_{T} .
$$


2.3. The cut-off problem corresponding to (1.1)-(1.4) and its well-posedness. Next, we focus on the study of the $\varepsilon$-problem (1.1)-(1.4). Here we consider a more general variant of the problem given by:

$$
\begin{gathered}
\frac{\partial \mathbf{u}_{\varepsilon}}{\partial t}=\mathbf{f}\left(\mathbf{u}_{\varepsilon}, \mathbf{v}_{\varepsilon}, x, t\right), \quad \text { in } \quad(0, T) \times \Omega ; \quad \mathbf{u}_{\varepsilon}(0)=\mathbf{u}^{0}(x), x \in \Omega ; \\
\frac{\partial \mathbf{v}_{\varepsilon}}{\partial t}-\frac{1}{\varepsilon} \Delta \mathbf{v}_{\varepsilon}=\Phi\left(\mathbf{u}_{\varepsilon}, \mathbf{v}_{\varepsilon}, x, t\right), \quad \text { in } \quad(0, T) \times \Omega ; \\
\mathbf{v}_{\varepsilon}(0)=\mathbf{v}^{0}(x), x \in \Omega ; \quad \frac{\partial \mathbf{v}_{\varepsilon}}{\partial \nu}=0 \quad \text { in } \quad(0, T) \times \partial \Omega .
\end{gathered}
$$

Existence of a mild solution for a short time follows from the standard theory, see e.g. the textbook of Henry [9] and monograph [21].

Nevertheless, in fact discussing the well-posedness of the $\varepsilon$-problem (2.18)(2.20) is not needed. We will study the cut-off problem and show that its solutions satisfy (1.1)-(1.4). It will give a posteriori the well posedness for all times $T \leq T_{0}$, where $T_{0}$ is the existence time of the maximal solution for the shadow problem (2.1)-(2.2). Our plan will be achieved using an explicit decomposition of the solution.

REMARK 2.3. Note that the present strategy is different than one in [19], where an independent proof of the short time existence and uniqueness of solutions of $\varepsilon$-problem (2.18)-(2.20) was provided.

The information that the time existence interval for variational solutions of problem (2.18)-(2.20) is always greater or equal to the existence time interval for problem (2.1)-(2.2), will be useful in the error estimation in Theorem 2.10. Such estimate on the time span of the solutions to the $\varepsilon$-problem was only conjectured in [19], but not proved.

We follow [19] and start by introducing the cut-off functions. The first cut-off function is

$$
\Theta(z)= \begin{cases}-\varepsilon \log (1 / \varepsilon), & \text { for } z<-\varepsilon \log (1 / \varepsilon) \\ z, & \text { for }-\varepsilon \log (1 / \varepsilon) \leq z \leq \varepsilon \log (1 / \varepsilon) \\ \varepsilon \log (1 / \varepsilon), & \text { for } z>\varepsilon \log (1 / \varepsilon) .\end{cases}
$$

Now we introduce the error functions by

$$
\mathcal{U}_{\varepsilon}=\mathbf{u}_{\varepsilon}-\mathbf{A} \text { and } \mathcal{V}_{\varepsilon}=\mathbf{v}_{\varepsilon}-\mathbf{B}-\varepsilon \mathbf{w}^{\Phi}-\xi^{i, \varepsilon} .
$$

Our goal is to estimate the error functions and to show that they are small in a suitable norm.

The function $\mathcal{V}_{\varepsilon}$ is given by

$$
\begin{gathered}
\frac{\partial \mathcal{V}_{\varepsilon}}{\partial t}-\frac{1}{\varepsilon} \Delta \mathcal{V}_{\varepsilon}=\Phi\left(\mathcal{U}_{\varepsilon}+\mathbf{A}, \mathcal{V}_{\varepsilon}+\mathbf{B}+\varepsilon \mathbf{w}^{\Phi}+\xi^{i, \varepsilon}, x, t\right)- \\
\Phi(\mathbf{A}, \mathbf{B}, x, t)-\varepsilon \partial_{t} \mathbf{w}^{\Phi} \quad \text { in } \quad \Omega \times(0, T) ; \\
\mathcal{V}_{\varepsilon}(x, 0)=0, x \in \Omega ; \quad \frac{\partial \mathcal{V}_{\varepsilon}}{\partial \nu}=0 \quad \text { in } \quad \partial \Omega \times(0, T) .
\end{gathered}
$$


Next, we write the right hand side in equation (2.23) as

$$
\begin{gathered}
\Phi\left(\mathcal{U}_{\varepsilon}+\mathbf{A}, \mathcal{V}_{\varepsilon}+\mathbf{B}+\varepsilon \mathbf{w}^{\Phi}+\xi^{i, \varepsilon}, x, t\right)-\Phi(\mathbf{A}, \mathbf{B}, x, t)= \\
\nabla_{A} \Phi(\mathbf{A}, \mathbf{B}, x, t) \mathcal{U}_{\varepsilon}+\nabla_{B} \Phi(\mathbf{A}, \mathbf{B}, x, t)\left(\mathcal{V}_{\varepsilon}+\varepsilon \mathbf{w}^{\Phi}+\xi^{i, \varepsilon}\right)+ \\
F\left(\mathcal{U}_{\varepsilon}, \mathcal{V}_{\varepsilon}+\varepsilon \mathbf{w}^{\Phi}+\xi^{i, \varepsilon}\right),
\end{gathered}
$$

where $F$ is quadratic in its variables. Following ideas of the center manifold theory (see e.g. [2]), we construct a convenient cut-off in $F$. We use the second cut-off function $\rho: \mathbb{R} \rightarrow[0,1]$, being a $C^{\infty}$ function with compact support and satisfying

$$
\rho(\zeta)= \begin{cases}1, & \text { for }|\zeta| \leq 1 \\ 0, & \text { for }|\zeta| \geq 2\end{cases}
$$

Next we set $\tilde{F}_{\varepsilon}(y, z)=\rho\left(\frac{|z|}{\sqrt{\varepsilon}}\right) F\left(\Theta\left(y_{1}\right), \ldots, \Theta\left(y_{n}\right), z\right)$. It is straightforward to see that

$$
\begin{gathered}
\left|\tilde{F}_{\varepsilon}(y, z)\right|=O(1)|(y, z)|^{2},\left|\frac{d}{d y} \tilde{F}_{\varepsilon}(y)\right|=O(1)|(y, z)|,\left\|\tilde{F}_{\varepsilon}\right\|_{C^{1}}=O(1) \sqrt{\varepsilon} \\
\left\|\tilde{F}_{\varepsilon}\right\|_{C}=O(1) \varepsilon .
\end{gathered}
$$

Our cut-off of the higher order terms in (2.25) is

$$
\begin{aligned}
F_{\varepsilon}(y, z, t) & =\rho\left(\frac{|z|}{\sqrt{\varepsilon}}\right) F\left(\Theta\left(y_{1}\right), \ldots, \Theta\left(y_{n}\right), z\right)\left(1-\rho\left(\frac{2 t \lambda_{1}}{-\varepsilon \log \varepsilon}\right)\right) \\
& +\rho\left(\frac{|z|}{2 C_{\xi}}\right) \rho\left(\frac{2 t \lambda_{1}}{-\varepsilon \log \varepsilon}\right) F\left(\Theta\left(y_{1}\right), \ldots, \Theta\left(y_{n}\right), z\right),
\end{aligned}
$$

where $C_{\xi}=2\left\|\mathbf{v}^{0}-\varepsilon \mathbf{w}^{\Phi}-\left\langle\mathbf{v}^{0}\right\rangle_{\Omega}\right\|_{L^{\infty}(\Omega)^{k}}$.

A direct calculation gives

LEMMA 2.4. There is a constant $C>0$, independent of $\varepsilon$, such that for all $(y, z, t)$ we have

$$
\left|F_{\varepsilon}(y, z)\right| \leq C \varepsilon+C \mathbb{1}_{\left\{t \leq-\varepsilon \log \varepsilon / \lambda_{1}\right\}} \min \{1,|z|\} .
$$

Next, we study the initial value problem for $\mathcal{U}_{\varepsilon}$, defined by $(2.22)$ :

$$
\begin{gathered}
\frac{\partial \mathcal{U}_{\varepsilon}}{\partial t}=\mathbf{f}\left(\mathbf{A}+\mathcal{U}_{\varepsilon}, \mathcal{V}_{\varepsilon}+\mathbf{B}+\right. \\
\left.\varepsilon \mathbf{w}^{\Phi}+\xi^{i, \varepsilon}, x, t\right)-\mathbf{f}(\mathbf{A}, \mathbf{B}, x, t) \text { in }(0, T) ; \mathcal{U}_{\varepsilon}(x, 0)=0,
\end{gathered}
$$

for almost all $x \in \Omega$. We write the nonlinearities at the right hand side in the following form:

$$
\begin{aligned}
\mathbf{f}\left(\mathbf{A}+\mathcal{U}_{\varepsilon}, \mathcal{V}_{\varepsilon}+\mathbf{B}\right. & \left.+\varepsilon \mathbf{w}^{\Phi}+\xi^{i, \varepsilon}, x, t\right)-\mathbf{f}(\mathbf{A}, \mathbf{B}, x, t)= \\
\nabla_{A} \mathbf{f}(\mathbf{A}, \mathbf{B}, x, t) \mathcal{U}_{\varepsilon} & +\nabla_{B} \mathbf{f}(\mathbf{A}, \mathbf{B}, x, t)\left(\mathcal{V}_{\varepsilon}+\varepsilon \mathbf{w}^{\Phi}+\xi^{i, \varepsilon}\right) \\
& +\mathbf{G}\left(\mathcal{U}_{\varepsilon}, \mathcal{V}_{\varepsilon}+\varepsilon \mathbf{w}^{\Phi}+\xi^{i, \varepsilon}\right),
\end{aligned}
$$


where $\mathbf{G}$ is quadratic in its arguments. As before, we will slightly modify arguments in $\mathbf{G}$ and consider the function $\mathbf{G}_{\varepsilon}$ given by

$$
\begin{aligned}
\mathbf{G}_{\varepsilon}(y, z) & =\rho\left(\frac{|z|}{\sqrt{\varepsilon}}\right) \mathbf{G}\left(\Theta\left(y_{1}\right), \ldots, \Theta\left(y_{n}\right), z\right)\left(1-\rho\left(\frac{2 t \lambda_{1}}{-\varepsilon \log \varepsilon}\right)\right) \\
& +\rho\left(\frac{|z|}{2 C_{\xi}}\right) \rho\left(\frac{2 t \lambda_{1}}{-\varepsilon \log \varepsilon}\right) \mathbf{G}\left(\Theta\left(y_{1}\right), \ldots, \Theta\left(y_{n}\right), z\right) .
\end{aligned}
$$

In analogy with Lemma 2.4 there is a constant $C>0$, independent of $\varepsilon$, such that for all $(y, z)$ we have

$$
\left|\mathbf{G}_{\varepsilon}(y, z)\right| \leq C\left(\varepsilon+\min \{1,|z|\} \mathbb{1}_{\left\{t \leq-\varepsilon \log \varepsilon /\left(\lambda_{1}\right)\right\}}\right) .
$$

Note that taking care of the initial layer $\xi^{\varepsilon, i}$ in (2.32) guarantees that the $L^{1}$-norm of $G_{\varepsilon}$ is $O(\varepsilon)$.

Next, define the barrier functions $\left\{C^{M}, C^{\gamma}\right\}$ through the system

$$
\begin{aligned}
& \frac{d}{d t} C^{M}=\mu_{A} C^{\gamma}+\mu_{B} C^{M}+\mathcal{W}\left(C^{M}, t\right), \quad C^{M}(0)=0, \\
& \frac{d}{d t} C^{\gamma}=\nu_{A} C^{\gamma}+\nu_{B} C^{M}+\mathcal{C}\left(C^{M}, t\right), \quad C^{\gamma}(0)=0,
\end{aligned}
$$

where $\mu_{A}=\left\|\nabla_{A} \Phi(\mathbf{A}, \mathbf{B}, x, t)\right\|_{L^{\infty}\left(Q_{T}\right)}, \mu_{B}=\left\|\nabla_{B} \Phi(\mathbf{A}, \mathbf{B}, x, t)\right\|_{L^{\infty}\left(Q_{T}\right)}$, $\nu_{A}=\left\|\partial_{A} \mathbf{f}(\mathbf{A}, \mathbf{B}, x, t)\right\|_{L^{\infty}\left(Q_{T}\right)}$ and $\nu_{B}=\left\|\partial_{A} \mathbf{f}(\mathbf{A}, \mathbf{B}, x, t)\right\|_{L^{\infty}\left(Q_{T}\right)}$. After $(2.28)$ and $(2.32)$, we have

$$
\begin{gathered}
\left\|\mathbf{G}_{\varepsilon}\left(y, z+\varepsilon w^{\Phi}+\xi^{i, \varepsilon}\right)+\nabla_{B} \mathbf{f}(\mathbf{A}, \mathbf{B}, x, t)\left(\varepsilon w^{\Phi}+\xi^{i, \varepsilon}\right)\right\|_{L^{\infty}(\Omega)} \\
\leq C_{0}\left(\varepsilon+e^{-2 \lambda_{1} t / \varepsilon}+|z| \mathbb{1}_{\left\{t \leq-\varepsilon \log \varepsilon /\left(\lambda_{1}\right)\right\}}\right)=\mathcal{C}(z, t), \\
\left\|\mathbf{F}_{\varepsilon}\left(y, z+\varepsilon w^{\Phi}+\xi^{i, \varepsilon}\right)+\nabla_{B} \Phi(\mathbf{A}, \mathbf{B}, x, t)\left(\varepsilon w^{\Phi}+\xi^{i, \varepsilon}\right)\right\|_{L^{\infty}(\Omega)} \\
\leq C\left(\varepsilon+e^{-2 \lambda_{1} t / \varepsilon}+|z| \mathbb{1}_{\left\{t \leq-\varepsilon \log \varepsilon /\left(\lambda_{1}\right)\right\}}\right)=\mathcal{W}(z, t) .
\end{gathered}
$$

The above estimates define the functions $\mathcal{W}$ and $\mathcal{C}$.

The basic theory of the ordinary differential equations yields

$$
\left\|C^{\gamma}\right\|_{L^{\infty}(0, T)}+\left\|C^{M}\right\|_{L^{\infty}(0, T)} \leq C(T) \varepsilon .
$$

Finally, we introduce the vector cut-off function $\Theta_{0}$ defined by

$$
\left(\Theta_{0}\left(a_{i}\right)\right)_{i}= \begin{cases}C^{M}, & \text { if } a_{i}>C^{M} \\ a_{i}, & \text { if } C^{M}>a_{i}>-C^{M} ; \\ -C^{M}, & \text { if } a_{i}<C^{M}\end{cases}
$$

$i=1, \ldots, k$. 
Now we study the "cut off nonlinearities" system attached to (2.23), (2.24), $(2.29)$ :

$$
\begin{gathered}
\frac{\partial \beta_{\varepsilon}}{\partial t}-\frac{1}{\varepsilon} \Delta \beta_{\varepsilon}=\alpha(x, t) \gamma_{\varepsilon}+b(x, t) \beta_{\varepsilon}+\mathbf{F}_{0}(x, t)+\mathbf{F}_{\varepsilon} \text { in } \Omega \times(0, T) \\
\beta_{\varepsilon}(x, 0)=0, x \in \Omega ; \quad \frac{\partial \beta_{\varepsilon}}{\partial \nu}=0 \quad \text { on } \quad \partial \Omega \times(0, T) \\
\frac{\partial \gamma_{\varepsilon}}{\partial t}=\mathcal{A}(x, t) \gamma_{\varepsilon}+\mathcal{A}_{0}(x, t) \beta_{\varepsilon}+\mathbf{G}_{0}(x, t)+\mathbf{G}_{\varepsilon} \text { in } \Omega \times(0, T) \\
\gamma_{\varepsilon}(x, 0)=0, x \in \Omega
\end{gathered}
$$

with

$$
\begin{gathered}
\alpha(x, t)=\nabla_{A} \Phi(\mathbf{A}, \mathbf{B}, x, t), \quad b(x, t)=\nabla_{B} \Phi(\mathbf{A}, \mathbf{B}, x, t), \\
\mathbf{F}_{0}(x, t)=b(x, t)\left(\varepsilon \mathbf{w}^{\Phi}+\xi^{i, \varepsilon}\right)-\varepsilon \partial_{t} \mathbf{w}^{\Phi}, \quad \mathcal{A}(x, t)=\nabla_{A} \mathbf{f}(\mathbf{A}, \mathbf{B}, x, t), \\
\mathbf{G}_{0}(x, t)=\nabla_{B} \mathbf{f}(\mathbf{A}, \mathbf{B}, x, t)\left(\varepsilon \mathbf{w}^{\Phi}+\xi^{i, \varepsilon}\right), \quad \text { and } \quad F_{\varepsilon}=F_{\varepsilon}\left(\gamma_{\varepsilon}, \beta_{\varepsilon}+\varepsilon \mathbf{w}^{\Phi}+\xi^{i, \varepsilon}\right) \\
\mathbf{G}_{\varepsilon}=\mathbf{G}_{\varepsilon}\left(\gamma_{\varepsilon}, \beta_{\varepsilon}+\varepsilon \mathbf{w}^{\Phi}+\xi^{i, \varepsilon}\right)+\nabla_{B} \mathbf{f}(\mathbf{A}, \mathbf{B}, x, t) \Theta_{0}\left(\beta_{\varepsilon}\right) .
\end{gathered}
$$

Note that all components of the matrices $\alpha, b$ and $\mathcal{A}$ are elements of $C^{1}([0, T] ; \mathcal{B}(\Omega))$. In equation $(2.38)$ we have $\mathcal{A}_{0}=0$, but the well-posedness result remains valid if it is an element of $C^{1}([0, T] ; \mathcal{B}(\Omega))^{\mathrm{km}}$.

We start by introducing the weak formulation for problem (2.36)-(2.39):

Find $\left\{\beta_{\varepsilon}, \gamma_{\varepsilon}\right\} \in L^{2}\left(0, T ; H^{1}(\Omega)^{k}\right) \cap C\left([0, T] ; L^{2}(\Omega)^{k}\right) \times H^{1}\left(0, T ; L^{2}(\Omega)^{m}\right)$, $\partial_{t} \beta_{\varepsilon} \in L^{2}((0, T) \times \Omega)^{k}$, and such that

$$
\begin{gathered}
\frac{d}{d t} \int_{\Omega} \beta_{\varepsilon}(t) \varphi d x+\frac{1}{\varepsilon} \int_{\Omega} \nabla \beta_{\varepsilon}(t): \nabla \varphi d x-\int_{\Omega} \alpha \gamma_{\varepsilon}(t) \varphi d x- \\
\int_{\Omega} b \beta_{\varepsilon}(t) \varphi d x=\int_{\Omega}\left(\mathbf{F}_{0}+\mathbf{F}_{\varepsilon}\left(\beta_{\varepsilon}(t), \gamma_{\varepsilon}(t), \cdot, \cdot\right)\right) \varphi d x, \quad \forall \varphi \in H^{1}(\Omega)^{k}, \\
\frac{d}{d t} \gamma_{\varepsilon}(x, t)-\mathcal{A} \gamma_{\varepsilon}(x, t)-\mathcal{A}_{0} \beta_{\varepsilon}(t)= \\
\mathbf{G}_{0}(x, t)+\mathbf{G}_{\varepsilon}\left(\beta_{\varepsilon}(x, t), \gamma_{\varepsilon}(x, t), x, t\right) \quad \text { a.e. on } \Omega \\
\beta_{\varepsilon}(0)=0 \text { and } \gamma_{\varepsilon}(x, 0)=\mathbf{0} \text { a.e. in } \Omega .
\end{gathered}
$$

Theorem 2.5. Under assumptions A1.-A2., A5., $\mathbf{F}_{0} \in L^{r}\left(Q_{T}\right)^{k}$ and $\mathbf{G}_{0} \in L^{r}\left(Q_{T}\right)^{m}, r>(n+2) / 2$, problem (2.40)-(2.42) admits a unique weak solution $\left\{\beta_{\varepsilon}, \gamma_{\varepsilon}\right\}$ on $\left(0, T_{0}\right)$. Furthermore, $\left\{\beta_{\varepsilon}, \gamma_{\varepsilon}\right\} \in W_{q}^{2,1}\left(Q_{T}\right)^{k} \times W^{1, q}\left(Q_{T}\right)^{m}$, $q<\min \{3, r\}$, for all $T \in\left(0, T_{0}\right)$.

Proof. The strategy of the proof is to first prove a short time existence and, then, to derive an priori estimate, allowing to conclude that the blow up never occurs and that a solution exists for any $T \leq T_{0}$, where $T_{0}$ is the existence time for the maximal solution to the shadow limit problem, defining $\{\mathbf{A}, \mathbf{B}\}$. 
For a short time existence we use Schauder's fixed point theorem. We take $R>0$ and set $B_{R}=\left\{f \in L^{2}((0, T) \times \Omega)^{k} \mid\|f\|_{L^{2}((0, T) \times \Omega)^{k}} \leq R\right\}$. Let $\beta^{*} \in B_{R}$.

We solve equation (2.41), with $\gamma(0)=0$ and $\beta_{\varepsilon}=\beta^{*}$. Note that the subscript $\varepsilon$ is dropped for simplicity. Since

$$
\partial_{t}|\gamma|^{2} \leq C_{1}|\gamma|^{2}+C_{2}\left|\mathcal{A}_{0} \beta^{*}+\mathbf{G}_{0}+\mathbf{G}_{\varepsilon}\right|^{2},
$$

Gronwall's inequality yields

$$
|\gamma|^{2}(x, t) \leq C e^{C_{1} t} \int_{0}^{t}\left(\left|\beta^{*}\right|^{2}+\left|\mathbf{G}_{0}\right|^{2}+\left|\mathbf{G}_{\varepsilon}\right|^{2}\right) d \tau .
$$

Hence

$$
\sup _{0 \leq t \leq T} \int_{\Omega}|\gamma(x, t)|^{2} d x \leq C(T)\left(C_{0}+R^{2}\right)
$$

and

$$
\int_{0}^{T} \int_{\Omega}|\gamma(x, t)|^{2} d x d t \leq T C(T)\left(C_{0}+R^{2}\right) .
$$

Next we solve equation (2.40), with $\beta(0)=0$ and $\gamma$ calculated above. After [13], chapter 4 , section 9 , it has a unique solution $\beta \in W_{q}^{2,1}\left(Q_{T}\right)^{k}, 1<q<$ $\min \{3, r\}$. The energy estimate reads

$$
\begin{gathered}
\int_{\Omega}|\beta|^{2}(t) d x+\frac{1}{\varepsilon} \int_{0}^{t} \int_{\Omega}|\nabla \beta|^{2} d x d \tau \leq C \int_{0}^{t} \int_{\Omega}|\gamma||\beta| d x d \tau \\
+C_{2} \int_{0}^{t} \int_{\Omega}|\beta|^{2} d x d \tau+\int_{0}^{t} \int_{\Omega}|\beta|\left(\left|\mathbf{F}_{0}\right|+\left|\mathbf{F}_{\varepsilon}\right|\right) d x d \tau \\
\leq C_{2} \int_{0}^{t} \int_{\Omega}|\beta|^{2} d x d \tau+C_{2} \int_{0}^{t} \int_{\Omega}|\gamma|^{2} d x d \tau \\
+C\left(\left\|\mathbf{F}_{0}\right\|_{L^{2}\left(Q_{T}\right)}^{2}+\left\|\mathbf{F}_{\varepsilon}\right\|_{L^{2}\left(Q_{T}\right)}^{2}\right) .
\end{gathered}
$$

Therefore, for $T \leq T_{\text {crit }}$ we have

$$
\|\beta\|_{L^{2}\left(Q_{T}\right)^{2}}^{2} \leq T \int_{\Omega}|\beta|^{2}(x, t) d x \leq T C(T)\left(1+R^{2}\right) \leq R^{2} .
$$

We set

$$
\mathcal{T} \beta^{*}=\beta
$$

Obviously, for $T \leq T_{\text {crit }}, \mathcal{T} B_{R} \subset B_{R}$. Also $\mathcal{T} B_{R} \subset W_{q}^{2,1}\left(Q_{T}\right)^{k}$ and it is a compact map. It remains to prove its continuity.

Let $\beta_{\ell}^{*} \rightarrow \beta^{*}$ in $B_{R}$, when $\ell \rightarrow+\infty$. Let $\gamma_{\ell}$ be the solution for equation (2.41), with $\gamma_{\ell}(0)=0$ and $\beta=\beta_{\ell}^{*}$. Then the substraction of the corresponding 
equations give

$$
\begin{gathered}
\frac{1}{2} \partial_{t} \int_{\Omega}\left|\gamma_{\ell}-\gamma\right|^{2}(t) d x=\int_{\Omega}\left(\mathcal{A}\left(\gamma_{\ell}-\gamma\right) \cdot\left(\gamma_{\ell}-\gamma\right)+\mathbf{A}\left(\beta_{\ell}^{*}-\beta^{*}\right) \cdot\left(\gamma_{\ell}-\gamma\right)+\right. \\
\left.\left(\mathbf{G}_{\varepsilon}\left(\gamma_{\ell}, \beta_{\ell}^{*}\right)-\mathbf{G}_{\varepsilon}\left(\gamma, \beta^{*}\right)\right)\left(\gamma_{\ell}-\gamma\right)\right) d x \leq C \int_{\Omega}\left|\gamma_{\ell}-\gamma\right|^{2}(t) d x+ \\
C \int_{\Omega}\left|\beta_{\ell}^{*}-\beta^{*}\right|^{2}(t) d x .
\end{gathered}
$$

Hence $\gamma_{\ell} \rightarrow \gamma$ in $L^{\infty}\left(0, T ; L^{2}(\Omega)^{m}\right)$.

Finally, let $\beta_{\ell}$ be the solution for equation $(2.40)$, with $\beta_{\ell}(0)=0$ and $\gamma=\gamma_{\ell}$. The Lipschitz property of $\mathbf{F}_{\varepsilon}$ with respect to $\gamma$ and $\beta$ and the basic parabolic theory, yields that

$$
\gamma_{\ell} \rightarrow \gamma \quad \text { in } \quad L^{\infty}\left(0, T ; L^{2}(\Omega)^{m}\right) \Rightarrow \beta_{\ell} \rightarrow \beta \quad \text { in } \quad W_{q}^{2,1}\left(Q_{T}\right)^{k}, \quad \text { as } \ell \rightarrow+\infty \text {. }
$$

Hence problem (2.40)-(2.42) has a weak solution for $T \leq T_{\text {crit }}$. Its uniqueness is obvious.

It remains to investigate what happens when $T$ reaches $T_{\text {crit }} \leq T_{0}$.

In order to prove that there is no explosion we need an a priori estimate. The a priori energy estimates are obtained by multiplying (2.40) by $\beta$ and integrating in time, then by multiplying (2.41) by $\gamma$ and integrating in time and, finally, by summing up both equalities. It yields

$$
\frac{1}{2} \int_{\Omega}\left(|\beta|^{2}(t)+|\gamma(t)|^{2}\right) d x+\frac{1}{\varepsilon} \int_{0}^{t} \int_{\Omega}|\nabla \beta(\tau)|^{2} d x d \tau=
$$

$\int_{0}^{t} \int_{\Omega}\left(\alpha \gamma(\tau) \cdot \beta(\tau)+b|\beta(\tau)|^{2}\right) d x+\int_{0}^{t} \int_{\Omega}\left(\mathbf{F}_{0}+\mathbf{F}_{\varepsilon}(\gamma(\tau), \beta(\tau), \cdot, \cdot)\right) \cdot \beta(\tau) d x d \tau$

$$
+\int_{0}^{t} \int_{\Omega}\left(\mathcal{A} \gamma(\tau)+\mathcal{A}_{0} \beta(\tau)+\mathbf{G}_{0}+\mathbf{G}_{\varepsilon}(\gamma(\tau), \beta(\tau), \cdot, \cdot)\right) \cdot \gamma(\tau) d x d \tau .
$$

Since $F_{\varepsilon}$ and $\mathbf{G}_{\varepsilon}$ are globally Lipschitz and bounded functions and all other coefficients are bounded, equality (2.47) implies

$$
\begin{gathered}
\|\beta\|_{L^{\infty}\left(0, T ; L^{2}(\Omega)^{k}\right)}+\left\|\nabla_{x} \beta\right\|_{L^{2}((0, T) \times \Omega)^{n k}} \leq C, \\
\|\gamma\|_{L^{\infty}\left(0, T ; L^{2}(\Omega)^{m}\right)} \leq C .
\end{gathered}
$$

Next we test equation (2.41) by $\partial_{t} \gamma$. It yields

$$
\left\|\partial_{t} \gamma\right\|_{L^{2}((0, T) \times \Omega)^{m}} \leq C .
$$

We continue by testing equation $(2.40)$ by $\partial_{t} \beta$. Using estimates (2.48)-(2.49) gives

$$
\left\|\partial_{t} \beta\right\|_{L^{2}((0, T) \times \Omega)^{k}} \leq C .
$$

Estimation (2.49) for $\gamma$ allows using the parabolic regularity theory from [13], chapter 4 , section 9 , which yields the boundedness of $\beta$ in $W_{2}^{2,1}\left(Q_{T}\right)^{k} \subset$ 
$L^{10}\left(Q_{T}\right)$. Now we go back to the Cauchy problem $(2.38)-(2.39)$, which gives boundedness of $\gamma$ in $H^{1}\left(0, T ; L^{10}(\Omega)\right)^{m}$. After coming back to problem (2.40)(2.41), we conclude that $\beta$ is uniformly bounded in $W_{q}^{2,1}\left(Q_{T}\right)^{k}, q<\min \{3, r\}$, by a constant, which can explode only when $T \rightarrow T_{0}$.

Now let us suppose that for $T=T_{\text {blow }}<T_{0}$ the constructed solution blows up. It means that its regularity deteriorates and the solution does not remain in the same class of functions for $T>T_{\text {blow }}$. Using estimates (2.48)-(2.51), we find out that $\beta$ is uniformly bounded in $W_{q}^{2,1}\left(Q_{T_{\text {blow }}}\right)^{k}, q<\min \{3, r\}$. After Lemma 3.4, page 82 from [13], $\beta\left(T_{\text {blow }}\right) \in W^{2-2 / q}(\Omega)^{k}, 2<q<\min \{3, r\}$, and $\gamma\left(T_{\text {blow }}\right) \in L^{10}(\Omega)^{m}$. Next, at $T=T_{\text {blow }}$ we restart system (2.40)-(2.41) with the new initial values. On the same way as in the beginning of the proof, we apply Schauder's theorem and obtain existence of a unique weak solution for $T_{\text {blow }} \leq t \leq T_{\text {blow }}+\delta, \delta>0$. Note that again $\beta \in W_{q}^{2,1}, q<\min \{3, r\}$. It contradicts the blow-up at $T=T_{\text {blow }}$. This proves the theorem.

2.4. $L^{\infty}$-estimates for the cut-off problem. A straightforward manipulation with equation (2.38) yields

$$
\partial_{t}\left|\gamma_{\varepsilon}\right| \leq \nu_{B} C^{M}+\nu_{A}\left|\gamma_{\varepsilon}\right|+\mathcal{C}\left(C^{M}, t\right)
$$

which, because of equation (2.34), implies the estimate

$$
\left|\gamma_{\varepsilon}(x, t)\right| \leq C^{\gamma} \leq C \varepsilon
$$

Proposition 2.6. Let Assumptions A1)-A2) hold, with $\mathbf{u}^{0} \in W^{1, r}(\Omega)^{m}$ and $\mathbf{v}^{0} \in W^{2, r}(\Omega)^{k}, r>(n+2) / 2$. Then there exists a constant $C>0$, independent of $\varepsilon$, such that for $\varepsilon \leq \varepsilon_{0}$ we have

$$
\sup _{(x, t) \in \Omega \times(0, T)}\left|\beta_{\varepsilon}(x, t)\right|+\sup _{(x, t) \in \Omega \times(0, T)}\left|\gamma_{\varepsilon}(x, t)\right| \leq C \varepsilon .
$$

Proof. We modify the approach from [13], chapter 7 , section 2 , to prove $L^{\infty}$-estimates for weakly coupled parabolic system. Because of the smoothness of the solution to problem (2.40)-(2.42), $\frac{\beta_{\varepsilon}^{i}}{\left|\beta_{\varepsilon}\right|}\left(\left|\beta_{\varepsilon}(x, t)\right|-C^{M}(t)\right)_{+}$, where $C^{M}$ is given by equation(2.33), can be used as a test function for equation (2.40). Direct computations give

$$
\begin{gathered}
\partial_{t} \beta_{\varepsilon} \cdot \frac{\beta_{\varepsilon}}{\left|\beta_{\varepsilon}\right|}\left(\left|\beta_{\varepsilon}\right|-C^{M}(t)\right)_{+}=\partial_{t}\left(\left|\beta_{\varepsilon}\right|-C^{M}(t)\right)_{+}^{2}+\frac{d C^{M}}{d t}\left(\left|\beta_{\varepsilon}\right|-C^{M}(t)\right)_{+}, \\
\nabla \beta_{\varepsilon}: \nabla\left(\frac{\beta_{\varepsilon}}{\left|\beta_{\varepsilon}\right|}\left(\left|\beta_{\varepsilon}\right|-C^{M}(t)\right)_{+}\right)= \\
\sum_{i=1}^{k}\left|\nabla \beta_{\varepsilon}^{i}\right|^{2} \frac{\left(\left|\beta_{\varepsilon}\right|-C^{M}(t)\right)_{+}}{\left|\beta_{\varepsilon}\right|}+\left|\nabla\left(\left|\beta_{\varepsilon}\right|-C^{M}(t)\right)_{+}\right|^{2} \frac{C^{M}}{4\left|\beta_{\varepsilon}\right|^{3}}, \\
\left|\nabla_{A} \Phi \gamma_{\varepsilon} \cdot \frac{\beta_{\varepsilon}}{\left|\beta_{\varepsilon}\right|}\left(\left|\beta_{\varepsilon}\right|-C^{M}(t)\right)_{+}\right| \leq \mu_{A}\left|\gamma_{\varepsilon}(x, t)\right|\left(\left|\beta_{\varepsilon}\right|-C^{M}(t)\right)_{+},
\end{gathered}
$$




$$
\begin{gathered}
\left|\nabla_{B} \Phi \beta_{\varepsilon} \cdot \frac{\beta_{\varepsilon}}{\left|\beta_{\varepsilon}\right|}\left(\left|\beta_{\varepsilon}\right|-C^{M}(t)\right)_{+}\right| \leq \mu_{B}\left(C^{M}(t)+\right. \\
\left.\left(\left|\beta_{\varepsilon}\right|-C^{M}(t)\right)_{+}\right)\left(\left|\beta_{\varepsilon}\right|-C^{M}(t)\right)_{+}, \\
\left|\left(\mathbf{F}_{0}+\mathbf{F}_{\varepsilon}\right) \cdot \frac{\beta_{\varepsilon}}{\left|\beta_{\varepsilon}\right|}\left(\left|\beta_{\varepsilon}\right|-C^{M}(t)\right)_{+}\right| \leq\left|\mathbf{F}_{0}+\mathbf{F}_{\varepsilon}\right|\left(\left|\beta_{\varepsilon}\right|-C^{M}(t)\right)_{+} .
\end{gathered}
$$

It yields the variational inequality

$$
\begin{gathered}
\frac{1}{2} \frac{d}{d t} \int_{\Omega}\left(\left|\beta_{\varepsilon}\right|-C^{M}(t)\right)_{+}^{2} d x+\frac{1}{\varepsilon} \int_{\Omega}\left|\nabla\left(\left|\beta_{\varepsilon}\right|-C^{M}(t)\right)_{+}\right|^{2} d x+ \\
\left.\int_{\Omega}\left(\left|\beta_{\varepsilon}\right|-C^{M}(t)\right)_{+}\left(\frac{d}{d t} C^{M}-\mu_{B} C^{M}-\mu_{A}\left|\gamma_{\varepsilon}\right|-\left|\mathbf{F}_{\varepsilon}+\mathbf{F}_{0}\right|\right)\right) d x \leq \\
\int_{\Omega} C\left(\left|\beta_{\varepsilon}\right|-C^{M}(t)\right)_{+}^{2} d x .
\end{gathered}
$$

By equation (2.33), $C^{M}$ is chosen in the way that the third term at the left hand side of (2.55) is nonnegative. Hence (2.37) and Gronwall's inequality give $\left(\left|\beta_{\varepsilon}\right|-C^{M}(t)\right)_{+}=0$ a.e. on $\Omega \times(0, T)$, i.e. $\left|\beta_{\varepsilon}(x, t)\right| \leq C^{M}(t)$ a.e. on $\Omega \times(0, T)$.

Now estimates (2.35) and (2.52) yield (2.54).

2.5. The error estimates and the shadow limit for the original system.

Proposition 2.7. For $\varepsilon \leq \varepsilon_{0}$, problem (2.23)-(2.24), (2.29) has a unique solution $\mathcal{U}_{\varepsilon}=\gamma_{\varepsilon}$ and $\mathcal{V}_{\varepsilon}=\beta_{\varepsilon}$, satisfying the $L^{\infty}$-error estimate

$$
\left\|\mathcal{U}_{\varepsilon}\right\|_{L^{\infty}(\Omega \times(0, T))^{m}}+\left\|\mathcal{V}_{\varepsilon}\right\|_{L^{\infty}(\Omega \times(0, T))} \leq C \varepsilon .
$$

Proof. Since for $\varepsilon \leq \varepsilon_{0}\left(\beta_{\varepsilon}, \gamma_{\varepsilon}\right)$ satisfies estimate (2.54), the cut-off operations in (2.27) and (2.31) do not change the nonlinearities. Hence we have $\mathbf{F}_{\varepsilon}=\mathbf{F}$ and $\mathbf{G}_{\varepsilon}=\mathbf{G}$. Consequently, the cut-off problem (2.36)-(2.39) and the $\varepsilon$-problem $(2.23)-(2.24),(2.29)$ are identical and $\left(\beta_{\varepsilon}, \gamma_{\varepsilon}\right)$ is a solution for problem (2.23)-(2.24), (2.29). By uniqueness of the smooth solutions, $\gamma_{\varepsilon}=\mathcal{U}_{\varepsilon}$ and $\beta_{\varepsilon}=\mathcal{V}_{\varepsilon}$. Estimate (2.54) implies (2.56).

It is convenient to decompose it to $\mathcal{V}_{\varepsilon}=\left\langle\mathcal{V}_{\varepsilon}\right\rangle_{\Omega}+\mathbf{H}_{\varepsilon},\left\langle\mathbf{H}_{\varepsilon}\right\rangle_{\Omega}=0$ and estimate both terms, $\left\langle\mathcal{V}_{\varepsilon}\right\rangle_{\Omega}$ and $\mathbf{H}_{\varepsilon}$, separately.

Using a constant as a test function in (2.23)-(2.24) and applying Gronwall's inequality yield

Corollary 2.8. Let $\left(\mathcal{U}_{\varepsilon}, \mathcal{V}_{\varepsilon}\right)$ be given by (2.29), (2.23)-(2.24). Then

$$
\left\|\left\langle\mathcal{V}_{\varepsilon}\right\rangle_{\Omega}\right\|_{L^{\infty}(0, T)} \leq C(T) \varepsilon \text {. }
$$

Next we estimate the perturbation term $\mathbf{H}_{\varepsilon}=\mathcal{V}_{\varepsilon}-\left\langle\mathcal{V}_{\varepsilon}\right\rangle_{\Omega}$.

Proposition 2.9. The perturbation term $\mathbf{H}_{\varepsilon}$ satisfies the estimate

$$
\left\|\mathbf{H}_{\varepsilon}\right\|_{L^{\infty}\left(0, T ; L^{2}(\Omega)^{k}\right)}^{2}+\frac{1}{\varepsilon}\left\|\nabla \mathbf{H}_{\varepsilon}\right\|_{L^{2}(\Omega \times(0, T))^{n k}}^{2} \leq C(T) \varepsilon^{2} .
$$


Proof. We use $\mathbf{H}_{\varepsilon}$ as a test function for equation (2.23). It yields a standard energy equality

$$
\begin{gathered}
\frac{1}{2} \frac{d}{d t} \int_{\Omega}\left|\mathbf{H}_{\varepsilon}\right|^{2} d x+\frac{1}{\varepsilon} \int_{\Omega}\left|\nabla \mathbf{H}_{\varepsilon}\right|^{2} d x \leq \\
\left\|\nabla_{B} \Phi\right\|_{\infty} \int_{\Omega}\left|\mathbf{H}_{\varepsilon}\right|^{2} d x+\int_{\Omega}\left(|| \nabla_{A} \Phi \|_{\infty}\left|\mathcal{U}_{\varepsilon}\right|+\varepsilon\left|\partial_{t} w^{\Phi}\right|+\right. \\
\left.\left\|\nabla_{B} \Phi\right\|_{\infty}\left(\left|\left\langle\mathcal{V}_{\varepsilon}\right\rangle_{\Omega}\right|+\varepsilon\left|w^{\Phi}\right|+\left|\xi^{i, \varepsilon}\right|\right)\right)\left|\mathbf{H}_{\varepsilon}\right| d x
\end{gathered}
$$

where the $L^{\infty}$-norm of the gradient is taken on a compact, determined by a neighborhood of $(\mathbf{A}, \mathbf{B})$ containing $\left(\mathbf{u}_{\varepsilon}, \mathbf{v}_{\varepsilon}\right)$, with $\varepsilon \leq \varepsilon_{0}$. We estimate integrals of products of various components of the approximation by $\mathbf{H}_{\varepsilon}$. The leading order terms are

$$
\begin{gathered}
\int_{\Omega} \sqrt{\varepsilon}\left|\xi^{i, \varepsilon}\right| \frac{\left|\mathbf{H}_{\varepsilon}\right|}{\sqrt{\varepsilon}} d x \leq \frac{C_{0}}{\varepsilon} \int_{\Omega}\left|\mathbf{H}_{\varepsilon}\right|^{2} d x+C_{1} \varepsilon \int_{\Omega}\left|\xi^{i, \varepsilon}\right|^{2} d x \text { and } \\
\int_{\Omega} \sqrt{\varepsilon}\left|\mathcal{U}_{\varepsilon}\right| \frac{\left|\mathbf{H}_{\varepsilon}\right|}{\sqrt{\varepsilon}} d x \leq \frac{C_{0}}{\varepsilon} \int_{\Omega}\left|\mathbf{H}_{\varepsilon}\right|^{2} d x+C_{1} \varepsilon \int_{\Omega}\left|\mathcal{U}_{\varepsilon}\right|^{2} d x \\
\leq \frac{C_{0}}{\varepsilon} \int_{\Omega}\left|\mathbf{H}_{\varepsilon}\right|^{2} d x+C_{1} \varepsilon^{3} .
\end{gathered}
$$

Next we take sufficiently small $C_{0}$ and apply Poincaré's inequality $\left\|\mathbf{H}_{\varepsilon}\right\|_{L^{2}(\Omega)} \leq C_{p}\left\|\nabla \mathbf{H}_{\varepsilon}\right\|_{L^{2}(\Omega)}$ in the energy estimate. It yields

$$
\frac{d}{d t} \int_{\Omega}\left|\mathbf{H}_{\varepsilon}\right|^{2} d x+\frac{1}{\varepsilon} \int_{\Omega}\left|\nabla \mathbf{H}_{\varepsilon}\right|^{2} d x \leq C \varepsilon^{3}+C \varepsilon \int_{\Omega}\left|\xi^{i, \varepsilon}\right|^{2} d x .
$$

After integrating in time from 0 to $t$ and using the decay in time of $\xi^{i, \varepsilon}$, we obtain the assertion of the Proposition.

Our result is summarized in the shadow limit theorem.

TheOREM 2.10. Under Assumptions A1-A2, with $\mathbf{u}^{0} \in W^{1, r}(\Omega)^{m}$ and $\mathbf{v}^{0} \in W^{2, r}(\Omega)^{k}, r>(n+2) / 2$, it holds

$$
\begin{gathered}
\left\|\mathbf{u}_{\varepsilon}-\mathbf{A}\right\|_{L^{\infty}(\Omega \times(0, T))^{m}} \leq C(T) \varepsilon, \\
\left\|\left\langle\mathbf{v}_{\varepsilon}\right\rangle_{\Omega}-\mathbf{B}\right\|_{L^{\infty}(0, T)^{k}} \leq C(T) \varepsilon, \\
\sqrt{\varepsilon}\left\|\mathbf{v}_{\varepsilon}-\xi^{i, \varepsilon}-\mathbf{B}\right\|_{L^{\infty}(\Omega \times(0, T))^{k}}+ \\
\left\|\nabla\left(\mathbf{v}_{\varepsilon}-\xi^{i, \varepsilon}-\varepsilon \mathbf{w}^{\Phi}\right)\right\|_{L^{2}(\Omega \times(0, T))^{n k}} \leq C(T) \varepsilon^{3 / 2}
\end{gathered}
$$

on every time existence interval $(0, T)$ for problem (2.1)-(2.2), i.e. the maximal time existence interval for the shadow problem.

Proof. The proof of Theorem 2.10 is a direct consequence of Corollary 2.8 and Proposition 2.9. 
ACKNOWLEDGEMENTS.

The research of A. M. was partially supported by the LABEX MILYON (ANR-10-LABX-0070) of Université de Lyon, within the program "Investissements d'Avenir" (ANR-11-IDEX-0007) operated by the French National Research Agency (ANR). The research visits of A. M. to the IWR, Universität Heidelberg, in 2016-2017, were partially supported by the post-Romberg professorship program at The Heidelberg Graduate School HGS MathComp, IWR, Universität Heidelberg.

\section{REFERENCES}

[1] A. Bobrowski, Singular perturbations involving fast diffusion, J. Math. Anal. Appl. 427 (2015), 1004-1026.

[2] J. Carr, Applications of Centre Manifold Theory, Springer- Verlag, New York, 1981.

[3] L. Y. Chen, N. Goldenfeld and Y. Oono, Renormalization group theory for global asymptotic analysis, Phys. Rev. Lett. 73 (1994), 1311-1315.

[4] H. Chiba, $C^{1}$ approximation of vector fields based on the renormalization group method, SIAM J. Appl. Dyn. Syst. 7 (2008), 895-932.

[5] P. Gray and S. K. Scott, Autocatalytic reactions in the isothermal continuous stirred tank reactor: isolas and other forms of multistability, Chem. Eng. Sci. 38 (1983), $29-43$.

[6] R. E. Lee DeVille, A. Harkin, M. Holzer, K. Josić and T. Kaper, Analysis of a renormalization group method and normal form theory for perturbed ordinary differential equations, Phys. D 237 (2008), 1029-1052.

[7] A. Gierer and H. Meinhardt, A theory of biological pattern formation, Kybernetik 12 (1972), 30-39.

[8] J. K. Hale and K. Sakamoto, Shadow systems and attractors in reaction-diffusion equations, Appl. Anal. 32 (1989), 287-303.

[9] D. Henry, Geometric Theory of semilinear Parabolic Equations, Lecture Notes in Math. 840, Springer-Verlag, 1981

[10] S. Hock, Y. Ng, J. Hasenauer, D. Wittmann, D. Lutter, D. Trümbach, W. Wurst, N. Prakash and F. J. Theis, Sharpening of expression domains induced by transcription and microRNA regulation within a spatio-temporal model of mid-hindbrain boundary formation, BMC Syst Biol 7 (2013), 48.

[11] J. P. Keener, Activators and inhibitors in pattern formation, Studies in Applied Mathematics 59 (1978), 1-23.

[12] V. Klika, R. E. Baker, D. Headon and E. A. Gaffney, The influence of receptormediated interactions on reaction-diffusion mechanisms of cellular self-organization, Bull. Math. Biol. 74 (2012), 935-957.

[13] O. A. Ladyzenskaja, V. A. Solonnikov and N. N. Ural'ceva, Linear and Quasi-linear Equations of Parabolic Type, Translations of Mathematical Monographs Reprint Vol. 23, American Mathematical Society, Providence, RI, 1968.

[14] I. Lengyel and I. R. Epstein, A chemical approach to designing Turing patterns in reaction-diffusion systems, Proc. Natl. Acad. Sci. USA 89 (1992), 3977-3979.

[15] A. Marciniak-Czochra, S. Härting, G. Karch and K. Suzuki, Dynamical spike solutions in a nonlocal model of pattern formation, arXiv:1307.6236 (2013).

[16] A. Marciniak-Czochra, G. Karch and K. Suzuki, Unstable patterns in reactiondiffusion model of early carcinogenesis, J. Math. Pures Appl. 99 (2013), 509-543. 
[17] A. Marciniak-Czochra and M. Kimmel, Modelling of early lung cancer progression: influence of growth factor production and cooperation between partially transformed cells, Math. Models Methods Appl. Sci. 17 (2007), suppl., 1693-1719.

[18] A. Marciniak-Czochra and M. Kimmel, Reaction-diffusion model of early carcinogenesis: the effects of influx of mutated cells, Math. Model. Nat. Phenom. 3 (2008), 90-114.

[19] A. Marciniak-Czochra and A. Mikelić, Shadow limits via the renormalization group method and the center manifold method, Vietnam J. Math. 45 (2017), 103-125.

[20] J. D. Murray, Mathematical biology II. Spatial models and biomedical applications. 3rd edition, Interdisciplinary Applied Mathematics 18, Springer-Verlag, New York, 2003.

[21] F. Rothe, Global Solutions of Reaction-Diffusion Systems, Lecture notes in mathematics 1072, Springer-Verlag, Berlin, 1984.

\section{"Shadow" limes za sisteme paraboličkih i običnih diferencijalnih jednadžbi kroz rezanje}

\section{Anna Marciniak-Czochra i Andro Mikelić}

SAŽETAK. "Shadow" limes (limes kada difuzijski koeficijent teži u beskonačnost) je proučavan za sistem običnih diferencijalnih jednadžbi vezan s dijagonalnim sistemom semi-linearnih jednadžbi topline u ograničenom području s homogenim Neumannovim rubnim uvjetima. Skorašnji dokaz konvergencije kroz energetski pristup iz članka [19], razvijen za slučaj samo jedne parcijalne diferencijalne jednažbe, preinačen je i poopćen na slučaj vezanog sistema. Dan je novi dokaz konvergencije, koji se zasniva na proučavanju odgovarajućeg odrezanog sistema i na konstrukciji barijernih i usporednih test funkcija, novih u literaturi. Dokaz daje $L^{\infty}$-ocjenu greške, proporcionalnu inverzu koeficijenta difuzije.

Anna Marciniak-Czochra

Institute of Applied Mathematics, IWR and BIOQUANT

University of Heidelberg, Im Neuenheimer Feld 267,

69120 Heidelberg, Germany

E-mail: anna.marciniak@iwr.uni-heidelberg.de

Andro Mikelić

Univ Lyon, Université Claude Bernard Lyon 1, CNRS UMR 5208,

Institut Camille Jordan, 43 blvd. du 11 novembre 1918,

F-69622 Villeurbanne cedex, France

E-mail: andro.mikelic@univ-lyon1.fr

Received: 31.3.2017.

Revised: 26.5.2017. 